\title{
An Investigation of High Frequency Wide Band Low Noise Amplifier for Antenna Testing System
}

\author{
Muhammad Asif \\ Zakariyya \\ SPEC Energy DMCC, \\ Islamabad, Pakistan
}

\author{
Farman Ullah \\ Department of Electrical \\ Engineering COMSATS \\ Institute of Information \\ Technology, Wah, Pakistan
}

\author{
Muhammad Farhan \\ University of Science and \\ Technology, Bannu, \\ Pakistan
}

\begin{abstract}
:
A design of wide band low noise amplifier for a typical antenna system is investigated here. This utilizes a wide frequency band from 1-6 GHz .The design objective is to achieve minimum Noise Figure at reasonable gain throughout the entire band. The transistor model used is ATF58143, An e pHEMTfamily member offering the best noise performance. After simulations a NF below $1 \mathrm{db}$ is achieved from $1-3.6 \mathrm{GHz}$ band along with a gain above $10 \mathrm{~dB}$. While below $2 \mathrm{~dB}$ noise figure is achieved between $3.6-5.6 \mathrm{GHz}$ but at this the gain achieved is above $7 \mathrm{~dB}$. The input and output matching networks for the circuit are designed using micro wave transmission lines and PCB used is PTFE board.
\end{abstract}

\section{INTRODUCTION:}

For proficient communication, the receiver needs to amplify the received signal so that it can be processed to recover the information. To achieve optimum performance the signal to noise ratio (SNR) should be as large as possible. The performance of normal amplifier is not satisfactory as it amplifies both the signal as well as noise components equally. So there comes need to design low noise amplifiers to amplify the signal while rejecting most of the noise band. These amplifiers are widely used in wireless communications.

The growth of semi-conductor technologies in RF micro Electronics has made it possible to develop cheap, small sized, more efficient in power-dissipation and noise rejection as well as user friendliness, applications for wireless communication system over the last two decades. These wireless systems and products resulted in increased utility, the commercial momentum of which has been fueling further investments in the field of integrated circuit design and technology. The resulting advancements in system capabilities has developed greater interest and receptiveness on the part of consumer leading to more applications anticipated and more spectrum be brought to support the wireless infrastructure.

LNA's can be found in almost every radio frequency and microwave receiving equipment which is utilized in commercial and military applications. As in cellular mobile phones and wireless Local Area Networks(WLAN), SIGNAL Interceptors are also used in Doppler RADARS.
A low Noise amplifier is an extraordinary family member of electronic amplifiers used frequently in the field of wireless communication systems. In case of wireless systems, the core task of LNA is to capture the received signal directly from the antenna and amplify this weak noisy signal in such a manner that its noise level is minimized to a certain threshold. In other words, gain of the amplifier is assumed to be kept to a certain desirable level in order to maintain lowest possible noise figure thus enabling enhanced spectrum for the support of wireless infrastructure.

\section{DESIGN METHODOLOGY}

\subsection{Low Noise Amplifier Design phases}

This section briefly discusses the basic principles and considerations used in the design and analysis of high frequency wide band Low Noise Amplifier. The stability, Gain and Noise figure issues would be discussed in details in the following section. However certain major points are as follow

- Basics of Low Noise Amplifier Transistor Specifications.

- Design Process in detail.

- Transistor Selection.

- Designing tool selection.

What will be the direction of efforts to achieve the desired goals within the specified period of time is demonstrated in the below given figure 


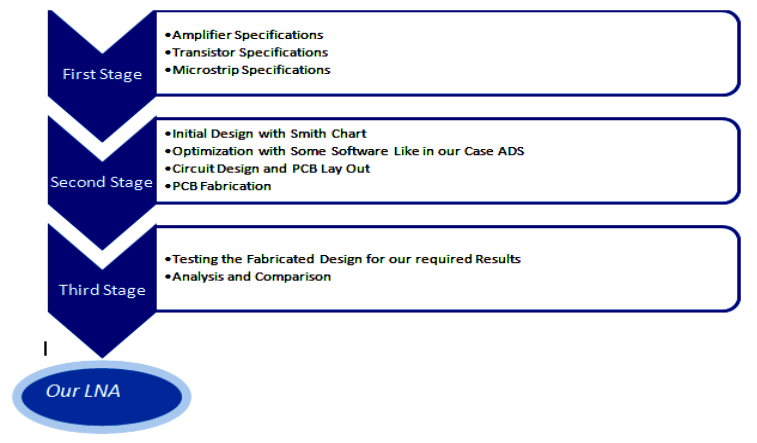

Figure 1: Design flow chart LNA design

\subsection{Design Parameters}

This section briefly looks at the design methodologies and considerations, involved in the formation of stable circuit design of Low noise amplifier and also discusses the constraints and tradeoffs between different parameters and technological limitations. For high speed data rates on the receiver circuit, the basic purpose of LNA is to provide lowest possible Noise figure and reasonable gain to render the sensitivity [1].It is mandatory to support higher order digital modulation formats. The principal focus of these designs is to achieve lowest possible noise figure by using PHEMT transistor technology for Antenna testing system. For antenna testing system the primary need only to get lowest possible noise figure of the pre-amplifier to amplify the weak signal that is being received by the antenna of the receiver [2][3]. Lowest possible noise figure will add very less amount of noise to it, which in the later stages results in minimum signal corruption in the demodulation process. The main objective is to strive for minimum possible noise figure of pre-amplifier capable of amplifying signals from 1 to $5 \mathrm{GHz}$, utilizing a wide band for antenna testing system [4]. The schematic is shown in the figure.

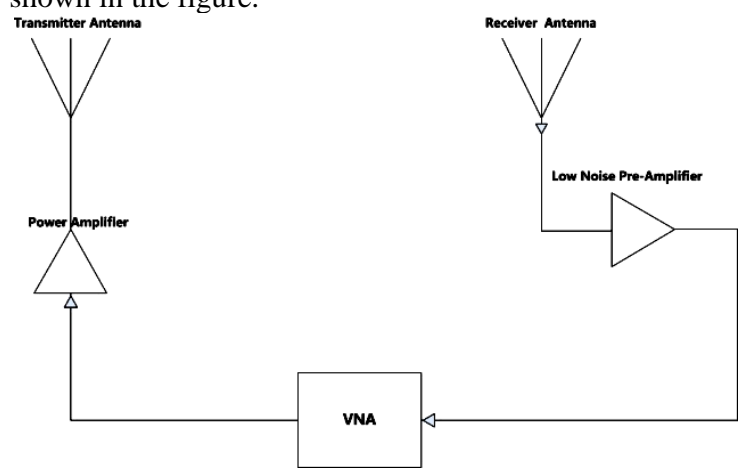

Figure 2: Testing of LNA with vector network analyzer

To prove this fact let us consider antenna testing demonstrated in the Fig where the $\mathrm{P}_{\mathrm{TRN}}$ is the power of transmitted signal from the transmitter, $\mathrm{G}_{\mathrm{PA}}$ is the gain of the power amplifier of the transmitter, $G_{A}$ is the gain of transmitter antenna, $G_{\text {prop }}$ is the losses received to it during propagation. While on the receiver side let $G_{A R}$ is the gain of receiver antenna and $\mathrm{G}_{\text {RFAMP }}$ is the gain of Low noise Pre amplifier installed just after the receiver antenna [5].

From propagation equation, the power of received signal is given by:

$P_{\text {power }}=P_{\text {trans }} * G_{P^{*}} G_{A^{*}} G_{\text {prop }} * G_{A R} * G_{\text {RFAMP }}$

Since Ptrans, $\mathrm{G}_{\mathrm{PA}}, \mathrm{G}_{\text {prop, }}, \mathrm{G}_{\mathrm{AR}}$ and $\mathrm{G}_{\mathrm{RFAMP}}$ are considered constant for an antenna system, the only dependence of the received power remains on the gain of the transmitter antenna $\mathrm{G}_{\mathrm{A}}$. So mathematically [6][7].
$\mathrm{K}=\operatorname{Ptrans} * \mathrm{G}_{\mathrm{PA}} * \quad \mathrm{G}_{\text {prop}} * \quad \mathrm{G}_{\mathrm{AR}} *$

So propagation equation becomes

$\mathrm{P}_{\text {power }}=\mathrm{K} * \mathrm{G}_{\mathrm{A}}$

In antenna system, the measurements are performed with respect to some known antenna [8]. So propagation equation for known antenna can be written as:

$\mathrm{P}_{\text {pknown }}=\mathrm{K}_{*} \mathrm{G}_{\text {Aknown }}$

While the measuring with the unknown antenna, the received power is [9][10]

$\mathrm{P}_{\mathrm{pu}}=\mathrm{K} * \mathrm{G}_{\text {Aunknown }}$

From equation 1 and 2

$$
\frac{\text { Ppknown }}{\text { Ppu }}=\frac{\text { GAknown }}{\text { GAunknown }}
$$

From the equation it is proved that gain of the LNA pre amplifier is not affecting the receiver performance. It is only the noise figure that matters in any antenna testing system. The design considerations are briefly discussed here:

\subsection{Stability Analysis}

The most important condition for an LNA is its stability. The stability of an amplifier is the ability to maintain its output characteristics in spite of large changes in the internal or external conditions such as physical temperature, operating frequency, source or load conditions etc. [11] In other words, it is the resistance of LNA to oscillate as oscillation is possible if either the input or output port impedance has a negative real part. This negative real part will cause the $\left|\Gamma_{\text {in }}\right|>$ 1 and $\left|\Gamma_{\text {out }}\right|>1 . \Gamma_{\text {in }}$ and $\Gamma$ out that are input and output reflection coefficients, depend upon the source and load matching networks, thus indirectly the stability of amplifier depends upon the $\Gamma_{\mathrm{s}}$ and $\Gamma_{\mathrm{L}}$ which are source and load reflection coefficients of the matching network respectively. [4][12]. Again s-parameters of a Low noise device, the matching networks and terminations all are used to determine whether the device will operate in stable mode or not.Two types of stabilities are usually encountered:

\section{- Unconditional Stability:}

The network is said to be unconditionally stable if the $\left|\Gamma_{\text {in }}\right|<1$ and $\left|\Gamma_{\text {out }}\right|<1$ for all passive source and load impedances. For this the magnitude of $\Gamma_{\mathrm{s}}$ and $\Gamma_{\mathrm{L}}$ must be less than one [13]. Unconditional stability of the circuit is the goal of LNA designer. In simple words unconditional stability means that with any load applied to the input or output of the device, the circuit will not oscillate and become unstable [14].

\section{- Conditional stability}

The device and network will be conditionally stable if the $\left|\Gamma_{\text {in }}\right|<1$ and $\left|\Gamma_{\text {out }}\right|<1$ only for a certain range of passive source and load impedances [4][12][15].Instabilities are primarily caused by three major factors:

- Internal feedback of the amplifying device

- $\quad$ External feedback around the transistor caused by external circuit 
- Excess of gain at frequencies that are outside of the band of the operation of the device

$\mathrm{S}$ parameters that are usually provided by the manufacturer are being used in the stability analysis of the Low noise amplifier circuit. Two major methodologies are employed in testing the stability. One is Rollet stability factor $\mathrm{K}$ that estimates the stability numerically while other is by plotting stability circles. Both will be discussed in detail.

Rollet Stability Test:It is also called K- $\Delta$ stability test. An intermitted quantity called $\Delta$ should be calculated first in order to determine the final value of $\mathrm{K}$. Both $\mathrm{K}$ and $\Delta$ are given in the next couple of equations [16]

$$
\begin{aligned}
K=\left(1-|\mathrm{S} 11|^{2}-|\mathrm{S} 22|^{2}\right. \\
\left.+|\Delta|^{2}\right) / 2|\mathrm{~S} 12 . \mathrm{S} 21| \\
|\Delta|=|\mathrm{S} 11 . \mathrm{S} 22-\mathrm{S} 12 . \mathrm{S} 21|^{2}
\end{aligned}
$$

For unconditional stability of the amplifier $|\mathrm{K}|>1$ and $|\Delta|<1$. These two simultaneous conditions are necessary and sufficient for the unconditional stability of the device [17]. If the device's s-parameters do not meet the above mentioned two conditions then conditional stability of the device is estimated by plotting the stability circles. With the help of these circles the designer estimates those values of $\Gamma \mathrm{s}$ and $\Gamma_{\mathrm{L}}$ that occurs in the stable region. For the device to be unconditionally stable, the values of $\left|S_{11}\right|$ and $\left|S_{22}\right|$ must have values less than unity.

\subsection{Stability Circles}

As discussed earlier these circles are used to estimate the stable regions for $\Gamma_{\mathrm{s}}$ and $\Gamma_{\mathrm{L}}$, when the device is conditionally stable. For a two port passive network, two types of stability circles are usually defined to locate the stable regions for both input and output matching networks.

- Input stability circle

- Output stability circle

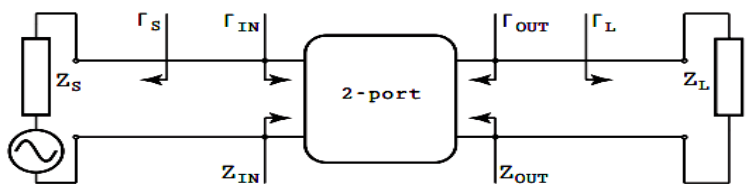

Figure 3: Stability of Two port network [4]

These two stability circles define the boundaries between the stable and unstable regions for different values of $\Gamma_{\mathrm{s}}$ and $\Gamma_{\mathrm{L}}$. The centre and radius of input stability circle is demonstrated in the below equations.

$\mathrm{C}_{\mathrm{in}}=\left(\mathrm{S}_{11}-\mathrm{S}_{22}{ }^{*} \Delta\right)^{*} /\left|\mathrm{S}_{11}\right|^{2}-|\Delta|^{2}$

$r_{\text {in }}=\left|\mathrm{S}_{12} \mathrm{~S}_{21}\right| /\left|\mathrm{iS}_{11} \mathrm{t}^{2}-\mathrm{l} \Delta \mathrm{t}^{2}\right|$

Where $C_{\text {in }}$ is center and $r_{\text {in }}$ is the radius of the input stability circle. Interchanging the values of $s_{11}$ and $s_{22}$ in the above equations will result in the output stability circle, center and radius expressions.

Consider the input stability circle drawn in $\Gamma \mathrm{s}$ plane for $\left|S_{22}\right|<1$ on left and $\left|S_{22}\right|>1$ on right in the figure. If $Z_{\mathrm{s}}=Z_{0}$, then $\Gamma_{\mathrm{S}}$ will become zero and this will result in $\left|\Gamma_{\text {out }}\right|=\left|S_{22}\right|$. If $\mid$ $\mathrm{S}_{22} \mid<1$ then definitely in $\left|\Gamma_{\text {out }}\right|$ would be less than unity and $\Gamma_{\mathrm{S}}$ $=0$ must occur in the stable region [18]. Since $\Gamma_{\mathrm{S}}=0$ in the centre of the smith chart hence the stable region must be inside the smith chart. Similarly if $\left|S_{22}\right|>1$, then $\left|\Gamma_{\text {out }}\right|>1$ and for $\Gamma_{\mathrm{S}}=0$, the centre of the smith chart should represent the unstable region. In this case the stable region will be the common region between the smith chart and the stability circle [4]. In a similar fashion this procedure can be applied to the output stability for locating stable circle regions.
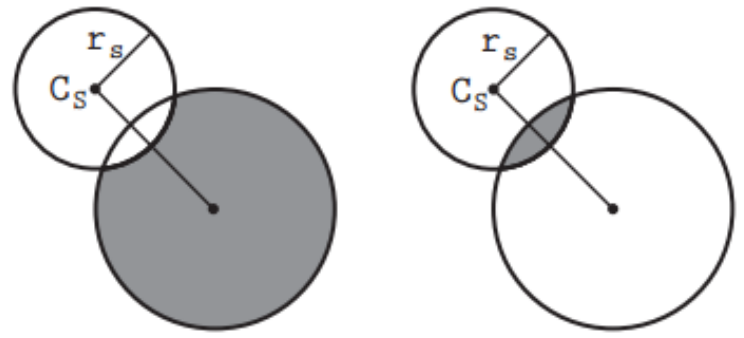

Figure 4: input stability circles in $\Gamma$ splane for a conditionally stable device. Left $|\mathrm{S} 22|<1$ and right $|\mathrm{S} 22|>1$ [11]

For unconditional case when both $\left|S_{11}\right|<1$ and $\left|S_{22}\right|<1$, both stability circles will occur either completely outside the smith chart or completely enclose the smith chart. Usually designers don't draw stability circles for unconditionally stable device. [4]

\subsection{Noise Figure analysis}

In active RF networks noise figure is the quantity used to measure and compare the noise contents in ideal and nonideal networks. Noise Figure is basically degradation in the signal to noise figure while the signal travels from the input port to the output port of the active network. Its numerical value is represented by the noise factor $F$ which is the ratio of input put port signal to noise ratio to the output port signal to noise ratio. While $\mathrm{F}$ is expressed in $\mathrm{dB}$ is called Noise figure. Thus the Noise figure is [19]

$\mathrm{NF}=10 \log \mathrm{F}$

\section{While F = input SNR / output SNR}

When considering several amplifiers in cascade, the overall noise figure $\mathrm{F}_{\mathrm{T}}$ of cascaded system can be determined by the expression given in the equation [19][20]

$F_{\text {total }}=F_{1}+\left(F_{2}-1\right) / G_{1}+\left(F_{3}-1\right) / G_{1} G_{2} \ldots$ (5) Where NF is the Noise Figure of individual entity and $\mathrm{G}_{\mathrm{n}}$ is the gain of each constituent amplifier of the Cascaded system.

The expression shows that noise figure of the first stage $F_{1}$ dominates the overall Noise performance in a typical receiver system that is why a pre amplifier of lowest possible Noise figure is introduced after antenna for better noise performance in most of the modern age receivers. Generally it is not possible to get both minimum noise figure and maximum gain for a Low noise amplifier, so a tradeoff is made between these parameters according to the requirement of the design. This tradeoff is made possible by creating different constant gain circles and Noise figure circles to select a usable tradeoff between between gain and noise figure.

\subsection{Noise Figure Circles:}

Consider the noise equation 


$$
\frac{|\Gamma s-\Gamma o p t|^{2}}{1-|\Gamma s|^{2}}=\frac{F-F \min }{4 r n}|1+\Gamma o p t|^{2}
$$

the equation relates noise figure to the source reflection coefficient $\Gamma_{\mathrm{s} .}$ the noise figure circles representing a specific noise figure value can be drawn on the source side of the smith chart at specific value of $\Gamma_{\mathrm{s}}$. When these circles are plotted with available gain circles, it makes us capable to estimate the required tradeoff between gain and noise figure [21][22].To calculate the Noise figure circle, define

NoiseFigureParameter $=\frac{F-F m i n}{4 r n} \mid 1+$ ropt $\left.\right|^{2}$

Re arrange the equation to get

$$
\left|\Gamma_{\mathrm{s}}-\mathrm{C}_{\mathrm{Fi}}\right|=\mathrm{r}_{\mathrm{FI}}
$$

Where

$$
r F i=\frac{1}{N i+1} \sqrt{N^{2}}\left(1-|\Gamma o p t|^{2}\right)(\text { Radius })
$$

And

$$
C F i=\frac{\Gamma o p t}{1+N i}
$$

Radius is a scalar while centre $\mathrm{C}_{\mathrm{Fi}}$ is a complex quantity.while $\mathrm{r}_{\mathrm{FI}}$ is zero at $\Gamma_{\mathrm{s}}=\Gamma_{\mathrm{opt}} . \Gamma_{\mathrm{opt}}$ is usually provided in the data sheet at specific frequency.the noise figure circle is shown in the following figures.

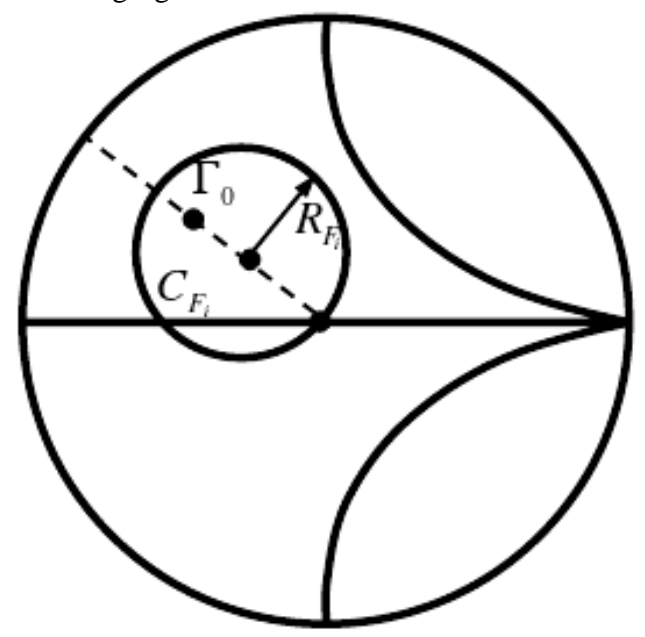

Figure 5: Noise Circles illustration

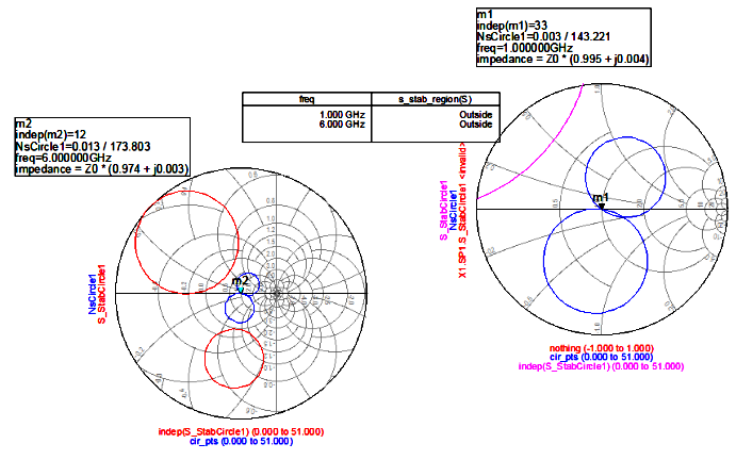

Figure 6: Stability Circles and Noise Figure Circles at 1 $\mathrm{GHz}$ and $6 \mathrm{GHz}$ with source stability circle.

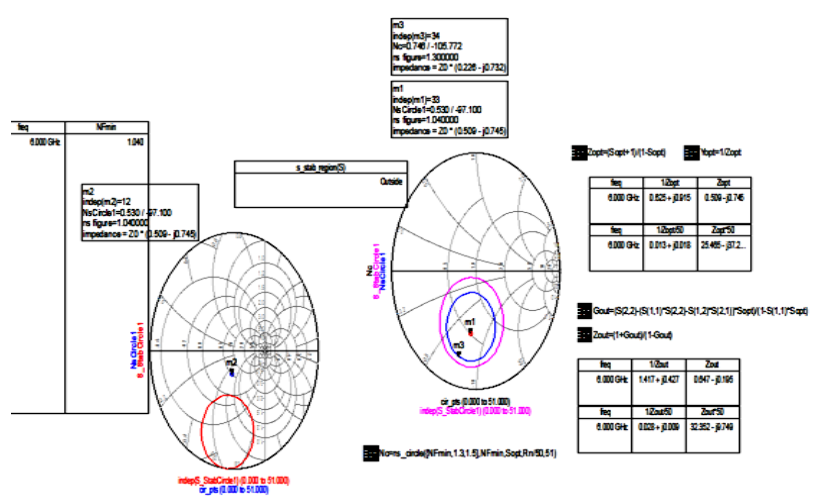

Figure 7: Estimation of noise figure by inserting different noise figure circles

The following table shows the noise parameters of ATF58143 PHEMT transistor IC at $\mathrm{V}_{\mathrm{DS}}=3$ Volts and $\mathrm{I}_{\mathrm{DS}}=10 \mathrm{~mA}$ bias point. This table reveals that as the operation of amplifier

\begin{tabular}{|c|c|c|c|c|c|}
\hline $\begin{array}{l}\text { Freq } \\
\text { GHz }\end{array}$ & $F_{\text {min }}$ & $\begin{array}{l}\mathrm{r}_{\text {opt }} \\
\text { Mag. }\end{array}$ & $\begin{array}{l}\mathbf{r}_{\text {opt }} \\
\text { Ang. }\end{array}$ & $\mathbf{R}_{\mathrm{n} S \mathrm{O}}$ & $\begin{array}{l}G_{a} \\
d B\end{array}$ \\
\hline 0.5 & 0.12 & 0.39 & 17.775 & 0.04 & 25.33 \\
\hline 0.9 & 0.18 & 0.37 & 46.9 & 0.04 & 22.26 \\
\hline 1.0 & 0.20 & 0.36 & 53.525 & 0.04 & 21.54 \\
\hline 1.5 & 0.32 & 0.32 & 80 & 0.04 & 19.16 \\
\hline 1.9 & 0.43 & 0.30 & 101 & 0.04 & 17.65 \\
\hline 2.0 & 0.45 & 0.30 & 107.7 & 0.04 & 17.33 \\
\hline 2.4 & 0.51 & 0.29 & 125.2 & 0.04 & 16.23 \\
\hline 3.0 & 0.58 & 0.31 & 154.475 & 0.05 & 14.77 \\
\hline 3.9 & 0.75 & 0.35 & -156.95 & 0.06 & 13.39 \\
\hline 5.0 & 0.87 & 0.42 & -120.93 & 0.09 & 11.92 \\
\hline 5.8 & 1.01 & 0.50 & -100.83 & 0.15 & 11.07 \\
\hline 6.0 & 1.04 & 0.53 & -97.15 & 0.18 & 10.93 \\
\hline
\end{tabular}
shoots to higher minimum noise figures $\mathrm{F}_{\text {min }}$, while dealing with higher frequencies.

Table 1: Typical noise Parameters

\subsection{Gain Considerations in Low Noise} Amplifier Design and Gain Circles:

A gain consideration in the design of a low noise amplifier plays an important role in the design process [23]. Thegain of a device is basically its ability to amplify the amplitude or power of the input signal [23]. It is the ratio of output to the input signal of the amplifying device,usually expressed in $\mathrm{dBs}$ voltageGain $=20 \log \left(6\left(\frac{\text { Vout }}{\text { Vin }}\right)\right.$ 
While power gain is generally defined as the ratio of the power actually delivered to the load to the power actually delivered by the source.[12][24]. A single stage microwave transistor low noise amplifier circuit consists of amplifying device like transistor and matching networks at both input and out put side of the transistor thepurpose of matching networks at input and output sides of the transistor is to transform the input and output impedence $Z_{0}$ to the source and load impedances $Z_{\mathrm{s}}$ and $\mathrm{Z}_{\mathrm{L}}$ [25].

Figure7 shows a two port network connected to the source $Z_{s}$ and load $Z_{L}$ impedance respectively.the derivation of expressions for three type of gain are discussed in the next lines. Here, mainly will be focused on three basic gain definitions while dealing with the low noise amplifier design. The most prominent is

- Transducer gain which is the average power delivered to the load to the maximum available average power from the source.

$$
\mathrm{G}_{\mathrm{T}}=\mathrm{P}_{\mathrm{L}} / \mathrm{P}_{\mathrm{AVS}}
$$

It is dependent upon both $\mathrm{Z}_{\mathrm{S}}$ and $\mathrm{Z}_{\mathrm{L}}$.

- Whileavailable power gain is defined as the ratio of maximum available average power at the load side to the maximum available average power from the source.

$$
\mathrm{G}_{\mathrm{A}}=\mathrm{P}_{\mathrm{AVN}} / \mathrm{P}_{\mathrm{AVS}}
$$

The maximum power gain can only be attained; if both input and output matching networks of the device are conjugate to each other. It depends upon $\mathrm{Z}_{\mathrm{s}}$ of the two port network. It is obvious that load and source reflection coefficients are given by

$$
\begin{aligned}
& \Gamma_{\mathrm{s}}=\mathrm{Z}_{\mathrm{S}^{-}} \mathrm{Z}_{\mathrm{O}} / \mathrm{Z}_{\mathrm{S}}+\mathrm{Z}_{\mathrm{O}} \quad \text { and } \quad \Gamma_{\mathrm{L}}=\mathrm{Z}_{\mathrm{L}^{-}} \mathrm{Z}_{\mathrm{O}} \\
& / \mathrm{Z}_{\mathrm{L}}+\mathrm{Z}_{\mathrm{O}}
\end{aligned}
$$

Where $\mathrm{Z}_{0}$ is the characteristic impedance reference for $\mathrm{s}$ parameters of the two port network.The expression for transducer gain is given by the below well-known equation.

$$
\begin{aligned}
& G T=\frac{P l}{P a v s}=|S 21|^{2}\left(1-|\Gamma s|^{2}\right)(1- \\
& \left.|\Gamma L|^{2}\right) /|| 1-\left.\Gamma s \Gamma i n\right|^{2}|1-S 22 \Gamma L|^{2}
\end{aligned}
$$

$\mathrm{G}_{\mathrm{T}}$ in terms multiplication of different gains is

$G T=\frac{1-|\Gamma s|^{2}}{|1-\Gamma i n \Gamma s|^{2}}|S 21|^{2} \frac{1-|\Gamma L|^{2}}{|1-S 22 \Gamma L|^{2}}=$

Gs. Go.GL

Where

$$
\begin{gathered}
G s=\frac{1-|\Gamma s|^{2}}{|1-\Gamma i n \Gamma s|^{2}} \\
G o=|S 21|^{2} \\
G L=\frac{1-|\Gamma L|^{2}}{|1-S 22 \Gamma L|^{2}}
\end{gathered}
$$

Where $\mathrm{G}_{\mathrm{S}}$ is the gain of input network, $\mathrm{G}_{\mathrm{O}}$ is the gain of transistor and $\mathrm{G}_{\mathrm{L}}$ is gain associated with the output matching network.

If $\Gamma_{\mathrm{s}}=\Gamma_{\mathrm{L}}=0$,

Then transducer gain FOR bilateral device becomes

$$
\mathrm{G}_{\mathrm{T}}=\left|\mathrm{S}_{21}\right|^{2}
$$

For unilateral case when $S_{12}=0$, the transducer gain will be

$$
\begin{aligned}
& \text { GTU } \\
& =\frac{1-|\Gamma s|^{2}}{|1-S 11 \Gamma s|^{2}}|S 21|^{2} \frac{1-|\Gamma L|^{2}}{|1-S 22 \Gamma L|^{2}}
\end{aligned}
$$

The maximum unilateral transducer gain $\mathrm{G}_{\mathrm{TU} \max }$ will be obtained, when both source and loads are conjugate of each other.

i.e. $\Gamma \mathrm{s}=\mathrm{S}_{11}^{*}, \mathrm{TL}=\mathrm{S}_{22}^{*}$,

Then

GTUmax $=\frac{1}{|1-S 11|^{2}}|S 21|^{2} \frac{1}{|1-S 22|^{2}}$

And $\mathrm{G}_{\mathrm{L}}, \mathrm{G}_{\mathrm{S}}$ and $\mathrm{Go}$ for maximum unilateral case becomes,

$$
\begin{aligned}
& G \text { smax }=\frac{1}{|1-S 11|^{2}} \\
& G \text { omax }=|S 21|^{2} \\
& G L \max =\frac{1}{|1-S 22|^{2}}
\end{aligned}
$$

These unilateral calculations are easy to solve if LNA designer solves the bilateral device with these easy unilateral expressions. First of all this will have to calculate the unilateral figure of merit in $\mathrm{dBs}$ first, which gives the estimation of the error caused due to application of unilateral expressions on a bilateral design given by the expression.

$$
\frac{1}{(1+U)^{2}}<\frac{G T}{G T U}<\frac{1}{(1-U)^{2}}
$$

Where ' $U$ ' is called unilateral figure of merits calculated from s-parameters.

$$
U=\frac{|S 11||S 21||S 12||S 22|}{(1-|S 11|)^{2}(1-|S 11|)^{2}}
$$

In order to draw constant gain circles, the radius and center of the circle for input matched network are given in the below couple of equations.

$$
\begin{aligned}
& \left.\mathrm{Cs}=\mathrm{gs} . \mathrm{S}^{*}{ }_{11} /\left[1-(1-\mathrm{gs})\left|\mathrm{S}_{11}\right|^{2}\right)\right] \\
& R s=\frac{\left[\sqrt{1-g s} \cdot\left(1-|S 11|^{2}\right)\right]}{1-(1-g s)|S 11|^{2}}
\end{aligned}
$$


Where $g_{s}$ is normalized gain factor for input matching network given $b_{g_{s}}=\mathrm{Gs} / \mathrm{Gs}$, $\max$. Similarly for output network constant gain circles just replace $s_{11}$ with $s_{22}$ for radius and centreexpressions. Thecenters of these constant gain circles are distributed along the lines from $\mathrm{S}_{11}{ }_{11}$ and $\mathrm{S}_{22}{ }_{22}$ from the center of smith chart. As shown in the figure

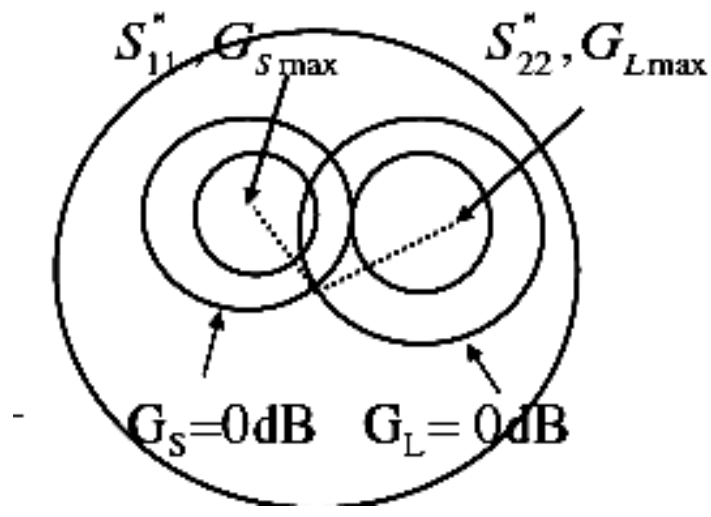

Figure 8: maximum gain occurs when point located at $\mathrm{s} 11 *$ and s22* in the $\Gamma$ s $\Gamma \mathrm{L}$ plane

\section{REFERENCES:}

[1] Lavasani, S.H.M.; Kiaei, S., "A new method to stabilize high frequency high gain CMOS LNA," Electronics, Circuits and Systems, 2003. ICECS 2003. Proceedings of the 2003 10th IEEE International Conference on, vol.3, no., pp.982,985 Vol.3, 14-17 Dec. 2003

[2] Alsunaidi, M.A.; El-Ghazaly, S.M., "High-frequency large-signal physical modeling of microwave semiconductor devices," Microwave Symposium Digest, 1995., IEEE MTT-S International, vol., no., pp.619,622 vol.2, 16-20 May 1995.

[3] K. Changed., Handbook of Microwave and optical Components,vol.2, wileyInterscience,N.Y.,1990

[4] Microwave engineering $3^{\text {rd }}$ edition by David M. Pozar

[5] A. Inoue, H. Amasuga, S. Watanabe, S. Goto, and T. Oku "MMIC technologies for MW and MMW applications" IEEE International Workshop on RadioFrequency Integration Technology, Dec. 2007.

[6] Gunel, T.; Yazgan, B., "A new approach to the design of low noise stable broadband microwave amplifier," Electrotechnical Conference, 1994. Proceedings., 7th Mediterranean, vol., no., pp.1150,1153 vol.3, 12-14 Apr 1994

[7] A. Tessmann, I. Kallfass, A. Leuther, H. Massler, M. Kuri, M. Riessle, M. Zink, R. Sommer, A. Wahlen, H. Essen, V. Hurm, M. Schlechtweg, and O. Ambacher "Metamorphic HEMT MMICs and Modules for Use in a High-Bandwidth $210 \mathrm{GHz}$ Radar" IEEE Journal Of Solid-State Circuits, Vol. 43, No. 10, October 2008.

[8] J. Sung, R. and S. Kim "Technology and Design Considerations for Millimeter-Wave Circuits" ICSICT 2008.

[9] Snowden, "Recent Development in Compound Semiconductor Microwave Power Transistor Technology", IEEE Proceedings Circuits, Devices \& Systems, Vol. 151, No. 3, June 2004.
[10] Nicholas J. Kolias, Colin S. Whelan, Thomas E. Kazior, and Kurt V. Smith "GaN Technology for Microwave and Millimeter-wave Applications" Raytheon Company, USA, IEEE 2010.

[11] Ji Li; Jixin Chen; Peng Chen; Fang Zhu, "VaractorTuned Half Mode Substrate Integrated Waveguide reflection-type phase shifter," Microwave Workshop Series on Millimeter Wave Wireless Technology and Applications (IMWS), 2012 IEEE MTT-S International vol., no., pp.1,3, 18-20 Sept. 2012

[12] MarcausEdwell,"Low-Noise Amplifier Design and Optimization", Luleả University of Technology 2008[13] Agilent ATF58143 data sheet low noise psedomorphic HEMT in a surface mount plastic package,www.alldatasheet.com

[13] Design and simulation of low noise amplifier circuit for 5 to $6 \mathrm{GHz}$,hosseinSahoolihzadehAlishirmoradi,world academy of science, engineering and technology 512009.

[14] Technology and design considerations for mili mere wave circuits,jae sung rieh

[15] Jae-Sung Rieh; Sooyeon Kim, "Technology and design considerations for millimeter-wave circuits," Solid-State and Integrated-Circuit Technology, 2008. ICSICT 2008. 9th International Conference on, vol., no., pp.1352,1356, 20-23 Oct. 2008

[16] J. D. Mellor and G. J. Linvill, "Synthesis of Interstage Networks ofPrescribed Gain Versus Frequency Slope," IEEE Transactions onMicrowave Theory and Techniques, vol. 23, no. 12, pp. 1013 - 1020,Dec. 1975.

[17] Wide band CMOS LNA for UHF applications, MSc dissertation by Ivy Iun lo sub,itted to university of HAWAI

[18] S.B.T Wang ,a sub mWatt 950MHz ultra wide band CMOS LNA,IEEE wireless communication symposium 2004.

[19] A. Bevilacqua and A. M. Niknejad, "An ultrawideband CMOS lownoiseamplifier for 3.1-10.6 GHz wireless receiver," IEEE J. Solid-StateCircuits, vol. 39, issue 12, pp. 2259 - 2268, Dec. 2004

[20] R. M. Fano, "Theoretical Limitations on the Broadband Matching ofArbitrary Impedances," Journal of Franklin Institute, Jan. 1950.

[21] Adriana Serban, "Dissertation on "Ultra-Wideband LowNoise Amplifier andSix-Port Transceiver for High Speed DataTransmission"

[22] Mohammad Afzalhussain, "low noise amplifier design for densedarrays",University of GAVLE, 2010

[23] H64RFP course work at moodle.nottingham.edu.my

[24] J Wiley, "Microwave circuit design using linear and nonlinear techniques",2010 